\title{
On the Locality Ideal in the Algebra of Test Functions for Quantum Fields
}

\author{
By \\ Jakob YNGVASON*
}

\begin{abstract}
Some basic properties of the locality ideal in Borchers's tensor algebra are established. It is shown that the ideal is a prime ideal and that the corresponding quotient algebra has a faithful Hilbert space representation. A topology is determined for which the positive cone in the quotient algebra is normal, and it is shown that every $n$-point distribution satisfying the locality condition is a linear combination of positive functionals which also satisfy that condition.
\end{abstract}

\section{§1. Introduction}

The locality ideal in Borchers' tensor algebra [1, 2] is the two-sided ideal generated by commutators of test functions with space-like separated supports. Its importance comes from the fact that quantum fields satisfying the requirement of local commutativity can be regarded as Hilbert space representations of the tensor algebra annihilating this ideal. Equivalently, such fields define representations of the corresponding quotient algebra. Among other things it will be shown that the states on this algebra separate points, and consequently that it has a faithful Hilbert space representation. For the tensor algebra itself this was first shown in [3]. The question whether this is also true for the quotient algebra was posed in [2], but has remained unsettled till now.

The present paper is a sequel to $[4,5,6]$, and we refer to these papers and also to [2] for definitions and further references. Borchers' tensor algebra will be denoted by $\underline{\mathscr{S}}$; it is the completed tensor algebra over the Schwartz space $\mathscr{Y}\left(\boldsymbol{R}^{d}\right)$. The locality ideal $\mathscr{F}_{c}$ is generated by elements of the form $f \otimes g$ $-g \otimes f$ with $f, g \in \mathscr{Y}\left(\boldsymbol{R}^{d}\right)$ satisfying the condition $f(x) g(y)=0$ whenever $(x-y)$ $\in \boldsymbol{R}^{d}$ is not space-like. The method used for the investigation of $\mathscr{F}_{c}$ and the

Communicated by H. Araki, December 15, 1983.

* The Science Institute, University of Iceland, 107 Reykjavik, Iceland. 
quotient algebra $\underline{\varphi} / \mathscr{F}_{c}=: A$ is based on a discretization: we map $\underline{\varphi}$ and $A$ onto tensor algebras over finite dimensional spaces where the corresponding problems are essentially of a combinatorial nature. Besides proving that the states on $A$ separate points, we also obtain more refined results analogous to Thms. 1 and 4 in [6] and Thm. 4.1 in [5]. We show that the positive cone $A^{+}$is normal for a certain topology which is slightly coarser than the Mackey topology on $A$. This result (Thm. 4.7) is not optimal. However, an explicit description of the finest topology on $A$ for which $A^{+}$is a normal cone seems to be complicated.

The paper is organized as follows: Section 2 contains general remarks on ideals in topological $*$-algebras. These remarks are mainly intended to show the equivalence of various definitions of "good" ideals and to point out the deviations from the case of $C^{*}$-algebras. In Section 3 we study partially symmetric tensor algebras over finitely dimensional spaces, i.e. tensor algebras with a finite number of generators, where some pairs of generators are assumed to commute. In Section 4 the results obtained for this discrete case are applied to the algebra $A$. The main results of the paper are contained in Thms. 4.44.7.

\section{§2. States and Ideals}

In this section we want to make some simple remarks on the ideals in $\underline{\varphi}$, or more generally, ideals in an arbitrary locally convex $*$-algebra $\mathfrak{A}$ over $\boldsymbol{C}$. We assume that the multiplication in $\mathfrak{A}$ is at least continuous in each factor separately, that the $*$-operation is continuous and that $\mathfrak{A}$ has a unit element 1. The positive cone $\mathfrak{\Re}^{+}$is defined as the closed convex hull of the set of elements of the form $a^{*} a, a \in \mathfrak{A}$. (For the algebras $\underline{\mathscr{S}}$ and $A$ this cone consists of convergent sums of such elements.) In the dual space $\mathfrak{X}^{\prime}$ the positive functionals form the dual cone $\mathfrak{Q}^{+\prime}$. A functional $T \in \mathfrak{A}^{+\prime}$ is a state if $T(1)=1$.

We associate three closed subspaces of $\mathfrak{A}$ with every $T \in \mathfrak{X}^{+\prime}$ :

The kernel $K(T)=\{a \mid T(a)=0\}$.

The left kernel $L(T)=\left\{a \mid T\left(a^{*} a\right)=0\right\}$.

The kernel of the GNS representation defined by $T$ :

$$
I(T)=\{a \mid a b \in L(T) \text { for all } b \in \mathfrak{X}\} .
$$

Clearly

$$
I(T) \subset L(T) \cap L(T)^{*} \subset \overline{L(T)+L(T)^{*}} \subset K(T) .
$$


By the Cauchy-Schwarz inequality we have also

$$
L(T)=\{a \mid b a \in K(T) \text { for all } b \in \mathfrak{U}\}
$$

and by polarization

$$
I(T)=\bigcap_{b} K\left(T_{b}\right)
$$

where $T_{b}(a):=T\left(b^{*} a b\right)$. From (2.1)-(2.3) we immediately get

2.1 Proposition. (i) $K(T)$ is *-invariant, and if $\sum_{1}^{\infty} a_{i}^{*} a_{i} \in K(T)$, then $\mathfrak{A}\left(a_{i}+a_{i}^{*} \mathfrak{A} \subset K(T)\right.$ for all $i$.

(ii) $L(T)$ is a left ideal, and if $\sum_{1}^{\infty} a_{i}^{*} a_{i} \in \overline{L(T)+L(T)^{*}}$, then $a_{i} \in L(T)$ for all $i$.

(iii) $I(T)$ is a *-invariant two-sided ideal, and $\sum_{i}^{\infty} a_{i}^{*} a_{i} \in I(T)$ implies $a_{i} \in I(T)$ for all $i$.

Also, $L(T)$ resp. $I(T)$ are the largest left-resp. two-sided ideals contained in $K(T)$.

By using the GNS-construction, the bipolar theorem and (2.3) one obtains

2.2 Proposition. The following conditions are equivalent for a closed, two-sided ideal $\mathscr{F} \subset \mathfrak{Q}$ :

(i) $\mathscr{F}$ is *-invariant and the quotient algebra $\mathfrak{A} / \mathscr{F}$ has a faithful Hilbert space representation ${ }^{1)}$.

(ii) $\mathscr{F}=\cap\left\{I(T) \mid T \in \mathscr{F}^{\perp} \cap \mathfrak{Q}^{+\prime}\right\}$.

(iii) $\mathscr{F}=\cap\left\{K(T) \mid T \in \mathscr{F}^{\perp} \cap \mathfrak{Q}^{+\prime}\right\}$.

In a $C^{*}$-algebra (more generally in an $L M C^{*}$-algebra [7]) every closed, two-sided ideal has these properties. Because of Prop. 2.1 a necessary condition for an ideal to have these properties is

$$
\sum_{1}^{\infty} a_{i}^{*} a_{i} \in \mathcal{F} \text { implies } a_{i} \in F^{F} \text { for all } i \text {. }
$$

From (2.4) the $*$-invariance of $\mathscr{F}$ follows: If $a \in \mathscr{F}$, then $a a^{*} \in \mathscr{F}$ because $\mathscr{F}$ is a right ideal. Hence $a^{*} \in F^{*}$ by (2.4).

In contrast to the $C^{*}$-algebra case, there are closed, two-sided ideals in $\underline{\varphi}$ which are not $*$-invariant, e.g. $\mathscr{F}=V \oplus\left(\bigoplus_{n=2}^{\infty} \mathscr{\varphi}_{n}\right)$ with $V \subset \mathscr{Y}_{1}$ closed and not *-invariant. Examples of $*$-invariant, two-sided ideals that violate (2.4) are $F=\bigoplus_{n=N}^{\infty} \mathscr{Y}_{n}$ with $N \geqq 2$, and the ideal generated by all anticommutators of ele-

1) i.e. a weakly continuous *-representation by linear operators (in general unbounded) on a dense domain in a Hilbert space. 
ments in $\mathscr{S}_{1}$. In these examples, $\mathscr{F}^{\perp} \cap \mathscr{\varphi}^{+\prime}$ consists only of trivial functionals. It is not known whether condition (2.4) characterizes the good ideals in $\underline{\varphi}$, i.e. those with the properties stated in Prop. 2.2.

\section{§3. Partially Symmetric Tensor Algebras}

Let $E$ be a finite dimensional vector space over $C$. Denote by $\underline{E}$ the tensor algebra over $E$, i.e.

$$
\underline{E}=\bigoplus_{n=0}^{\infty} E_{n}
$$

with $E_{0}=C, E_{n}=E^{\otimes n}$. A basis $\left\{e_{1}, \cdots, e_{N}\right\}$ for $E$ generates a basis for $\underline{E}$ : If $I=\left(i_{1}, \cdots, i_{n}\right), i_{k} \in\{1, \cdots, N\}$ we define

$$
e_{I}:=e_{i_{1}} \otimes \cdots \otimes e_{i_{n}}
$$

and also $e_{\phi}:=1 \in E_{0}$. We also write

$$
I^{*}:=\left(i_{n}, \cdots, i_{1}\right)
$$

and define a $*$-operation on $\underline{E}$ by antilinear extension of

$$
\left(e_{I}\right)^{*}:=e_{I^{*}}
$$

$\underline{E}$ is thus a $*$-algebra; equipped with the direct sum topology of the $E_{n} \simeq C^{n N}$ it becomes a topological $*$-algebra with a jointly continuous product.

Now suppose $\rho$ is an arbitrary set of pairs $(i, j)$ with $i, j \in\{1, \cdots, N\}$. Define $\mathcal{F}_{p}$ to be the two-sided ideal in $E$ generated by the commutators $\left[e_{i}, e_{j}\right]=$ $e_{i} \otimes e_{j}-e_{j} \otimes e_{i}$ with $(i, j) \in \rho$. Without restriction we can assume that $(i, i) \in \rho$ for all $i$ and that $(i, j) \in \rho$ implies $(j, i) \in \rho ; \rho$ is thus a symmetric, reflexive, but not necessarily transitive relation on $\{1, \cdots, N\}$.

The partially symmetric tensor algebra corresponding to $\rho$ is defined as the quotient algebra $\underline{E} / \mathscr{F}_{\rho}$.

Remark. The ideals $\mathscr{F}_{\rho}$ are generated by commutators of linearily independent elements in $E$. More generally one could consider ideals generated by arbitrary sets of commutators of elements in $E$. The latter case cannot always be reduced to the former by a linear transformation. A counterexample is e.g. provided by the set $\left[e_{1}, e_{2}\right],\left[e_{3}, e_{4}\right],\left[e_{5}, e_{2}+e_{4}\right]$ with $e_{1}, \cdots, e_{5}$ linearily independent. For the present purpose, however, the $\mathscr{F}_{\rho}^{\prime}$ 's defined above suffice.

The algebraic classification of partially symmetric tensor algebras of the kind considered here is for a given $N$ the same as the classification of symmetric, 
reflexive relations on $\{1, \cdots, N\}$. Such relations can be pictured by graphs with $N$ vertices. The number of non-isomorphic graphs and thus of algebraically distinct partially symmetric tensor algebras is a complicated expression and grows rapidly with $N$ [8].

$\underline{E} / \mathscr{F}_{\mathrm{p}}$ inherits the $*$-operation and the topology from $\underline{E}$. The dual space $\left(E / F_{\rho}\right)^{\prime}$ can be identified with $\mathscr{F}_{\rho}^{\perp} \subset \underline{E}^{\prime}$ and its positive part with $\underline{E}^{+\prime} \cap \mathcal{F}_{\rho}^{\perp}$. Since $\operatorname{dim} E<\infty$ every linear functional on $\underline{E}$ or $\underline{E} / \mathscr{F}_{\rho}$ is continuous.

It will be convenient to describe $\mathscr{F}_{\rho}$ and $\mathscr{F}_{\rho}^{\perp}$ in terms of the basis $\left\{e_{I}\right\}$. We define an equivalence relation for multiindices: Write $I \sim J$, or more precisely $I \widetilde{p} J$, if $I$ and $J$ can be transformed one into the other by a sequence of transpositions of adjacent pairs of indices in $\rho$, in other words, if $I=\left(i_{1}, \cdots, i_{n}\right)$, $J=\left(j_{1}, \cdots, j_{n}\right)$ and there is a finite sequence of multiindices $I_{\kappa}=\left(i_{1 \kappa}, \cdots, i_{n \kappa}\right)$, $\kappa=0, \cdots, k$ such that $I_{0}=I, I_{k}=J$ and $I_{\kappa}$ and $I_{\kappa+1}$ differ only in a single pair $\left(i_{r \kappa}, i_{r+1, \kappa}\right)=\left(i_{r+1, \kappa+1}, i_{r, \kappa+1}\right) \in \rho$.

3.1 Lemma. (i) Let $T \in \underline{E}^{\prime}$. Then $T \in \mathcal{F}_{\rho}^{\perp}$ iff $T\left(e_{I}\right)=T\left(e_{J}\right)$ for $I_{\widetilde{p}} J$. (ii) $\sum_{I} \alpha_{I} e_{I} \in \mathscr{F}_{\rho}$ iff $\sum_{I \tilde{\rho}^{J}} \alpha_{I}=0$ for all $J$.

Proof. (i) Suppose $I \sim J$ and let $I_{0}, \cdots, I_{k}$ be a sequence leading from $I$ to $J$. Then $e_{I}-e_{J}=\sum_{\kappa} e_{I_{\kappa}}-e_{I_{\kappa+1}}$ and clearly $T\left(e_{I}-e_{I_{\kappa+1}}\right)=0$ for $T \in \mathcal{F}_{\rho}^{\perp}$. The other direction is obvious.

(ii) The functionals

$$
T_{[J]}\left(e_{I}\right)= \begin{cases}1 & \text { if } I \in[J]:=\text { equivalence class of } J \\ 0 & \text { otherwise }\end{cases}
$$

form a basis for $\mathscr{F}_{\rho}^{\perp}$ by (i), and $\left(\mathscr{F}_{\rho}^{\perp}\right)^{\perp}=\mathscr{F}_{\rho}$.

The algebras $\underline{E}$ and $\underline{E} / \mathscr{F}_{\rho}$ have a natural grading. Equivalently we may talk about a grading of multiindices: If $I=\left(i_{1}, \cdots, i_{n}\right)$, we define

$$
\operatorname{Deg} I=\left(\nu_{1}, \cdots, \nu_{N}\right)
$$

where $\nu_{j} \in N \cup\{0\}$ counts how many times the index $j \in\{1, \cdots, N\}$ appears in I. Obviously

$$
\operatorname{Deg} I=\operatorname{Deg} I^{*} \text {. }
$$

If $I=\left(i_{1}, \cdots, i_{n}\right)$ and $J=\left(j_{1}, \cdots, j_{m}\right)$ we write

$$
I J=\left(i_{1}, \cdots, i_{n}, j_{1}, \cdots, j_{m}\right)
$$

and we have 


$$
\operatorname{Deg} I J=\operatorname{Deg} I+\operatorname{Deg} J \text {. }
$$

Note also that by lemma 3.1 (ii) we have that $\sum_{I} \alpha_{I} e_{I} \in \mathscr{F}_{\rho}$ implies $\sum_{\operatorname{Deg} I=\nu} \alpha_{I} e_{I} \in \mathscr{F}_{\rho}$ for all $\nu=\left(\nu_{1}, \cdots, \nu_{N}\right)$, i.e. $\mathcal{F}_{\rho}$ is a graded ideal with respect to this grading.

The following simple lemma is crucial for the subsequent considerations.

3.2 Lemma. If $I K \sim J L$ and $\operatorname{Deg} I=\operatorname{Deg} J$, then $I \sim J$ and $K \sim L$.

Proof. Let $(I K)_{\kappa}, \kappa=0, \cdots, k$ be a sequence of multiindices leading from $I K=(I K)_{0}$ to $J L=(I K)_{k}$ such that $(I K)_{\kappa}$ and $(I K)_{\kappa+1}$ differ only by an admissible transposition, i.e. a transposition of an adjacent pair in $\rho$. Clearly we need only consider such pairs $(i, j)$ with $i \neq j$, so we can uniquely link each index in $(I K)_{\kappa}$ to an index in $I$ or in $K$ by tracing back its "path" through the sequence of admissible transpositions. If $I=\left(i_{1}, \cdots, i_{n}\right)$, define $i_{1 \kappa}, \cdots, i_{n \kappa}$ to be those indices in $(I K)_{\kappa}$ which are linked to indices in $I$, numbered according to their position in $(I K)_{\kappa}$ from left to right. $I_{\kappa}:=\left(i_{1 \kappa}, \cdots, i_{n \kappa}\right)$ is then a permutation of $I$, and we contend that $I_{\kappa}$ and $I_{\kappa+1}$ differ at most by an admissible transposition. In fact, if the transposition leading from $(I K)_{k}$ to $(I K)_{k+1}$ does not affect the indices linked to $I$, or if it permutes an index linked to $I$ and an index linked to $K$, then $I_{\kappa}=I_{\kappa+1}$. If on the other hand it permutes two adjacent indices linked to $I$, then $I_{k}$ and $I_{k+1}$ differ by an admissible transposition. Hence $I_{\kappa} \sim I$ for all $\kappa$. Now because $\operatorname{Deg} I=\operatorname{Deg} J, J$ consists of the same indices as $I$. By our convention of never permuting two identical indices we conclude from $(I K)_{k}=J L$ that $J=I_{k} \sim I$. In the same way $K \sim L$.

As a last preparation we now order the degrees of multiindices, i.e. the $N$-tuples $\nu=\left(\nu_{1}, \cdots, \nu_{N}\right)$, by first ordering according to their length $\nu_{1}+\cdots+\nu_{N}$ and then lexicographically. In other words, we write

$$
\left(\nu_{1}, \cdots, \nu_{N}\right)<\left(\mu_{1}, \cdots, \mu_{N}\right)
$$

if either $\nu_{1}+\cdots+\nu_{N}<\mu_{1}+\cdots+\mu_{N}$, or the lengths are equal and there is a $k \in\{1, \cdots, N\}$ such that $\nu_{j}=\mu_{j}$ for $j=1, \cdots, k-1$ but $\nu_{k}<\mu_{k}$.

Note that

$$
\nu<\mu \text { and } \lambda \leqq \kappa \text { imply } \nu+\lambda<\mu+\kappa .
$$

If $a=\sum \alpha_{I} e_{I} \in \underline{E}, a \neq 0$, we define $\operatorname{Deg} a$ as the highest Deg $I$ such that $\alpha_{I} \neq 0$. Because of (3.2) and (3.3) we have

$$
\operatorname{Deg}(a b)=\operatorname{Deg} a+\operatorname{Deg} b
$$


provided $a, b \neq 0$. Using this ordering and Lemma 3.2 we can now establish some simple algebraic properties of $\mathscr{F}_{\rho}$.

3.3 Theorem. (i) The algebra $\underline{E} / \mathscr{F}_{\rho}$ has no divisors of zero.

(ii) The only invertible elements in $\underline{E} / \mathscr{F}_{\rho}$ are multiples of the identity.

(iii) If $\mathscr{B}$ is the subalgebra of $E / F^{\prime}$ generated by $e_{1}, \cdots, e_{k}, k \leq N$, then the commutant $\mathscr{B}^{\prime}$ is generated by the $e_{j}$ 's, such that $(i, j) \in \rho$ for $i=1, \cdots, k$.

Proof. (i) We have to show that if $a, b \in \underline{E}$ and $a b \in \mathscr{F}_{\rho}$, then either $a \in \mathscr{F}_{\rho}$ or $b \in \mathscr{F}_{\rho}$. Write $a=\sum \alpha_{I} e_{I}$ and $b=\sum \beta_{J} e_{J}$. Because of (3.3) the term of highest degree in $a b$ is

$$
\sum_{\substack{\operatorname{Deg} I=\nu \\ \operatorname{Deg} J=\mu}} \alpha_{I} \beta_{J} e_{I} e_{J}
$$

where $\nu=\operatorname{Deg} a, \mu=\operatorname{Deg} b$. Since $\mathscr{F}_{\mathrm{p}}$ is a graded ideal, this term belongs also to $\mathscr{F}_{\rho}$. By Lemma 3.1 (ii) we have therefore

$$
\sum_{\substack{I J \sim I_{0} J_{0} \\ \operatorname{Deg} I=\nu \\ \operatorname{Deg} I=\mu}} \alpha_{I} \beta_{J}=0
$$

for all $I_{0}, J_{0}$ with Deg $I_{0}=\nu, \operatorname{Deg} J_{0}=\mu$. Because of lemma 3.2 this means that

$$
\left(\sum_{I \sim J_{0}} \alpha_{I}\right)\left(\sum_{J \sim J_{0}} \beta_{J}\right)=0
$$

Now suppose $a \notin \mathscr{F}_{\rho}$ and $b \notin \mathscr{F}_{\rho}$. Since $a b \in \mathscr{F}_{\rho}$ and $\mathscr{F}_{\rho}$ is a two-sided ideal, we can subtract terms belonging to $\mathscr{F}_{\rho}$ from $a$ and $b$ if necessary and thus assume that the terms of highest degree in both $a$ and $b$ do not belong to $\mathscr{F}_{p}$. This contradicts (3.5) by Lemma 3.1 (ii).

(ii) The proof is essentially the same as in (i) with $\mathscr{F}_{p}$ replaced by $\mathscr{F}_{p}+\{\lambda \cdot 1\}$.

(iii) Suppose $a=\sum \alpha_{I} e_{I}$ commutes $\bmod \mathscr{F}_{\rho}$ with $e_{i}$. This means by lemma 3.1 (ii) that $i J \sim J i$ for all $J$ such that $\sum_{I \sim J} \alpha_{I} \neq 0$. Hence, $(i, j) \in \rho$ for all $j$ contained in $J$, so modulo $\mathscr{F}_{\rho} a$ belongs to the algebra generated by $e_{j}$ with $(i, j) \in \rho$.

We turn next to a construction of positive, linear functionals on $E / F_{\rho}$, or equivalently, functionals in $\mathscr{F}_{\rho}^{\perp} \cap \underline{E}^{+\prime}$. For every $T \in \mathscr{F}_{\rho}^{\perp} \cap \underline{E}^{+\prime}$ the matrix

$$
M_{I, J}:=T\left(e_{I}^{*} \otimes e_{J}\right)=T\left(e_{I^{*}}\right)
$$

has the following properties:

1) $M_{I, J}$ is positive semidefinite, i.e. 


$$
\sum \bar{\lambda}_{I} \lambda_{J} M_{I, J} \geq 0 \text { for all finite sequences }\left\{\lambda_{I}\right\} \text { in } \boldsymbol{C},
$$

2) If $I^{*} J \sim K^{*} L$, then $M_{I, J}=M_{K, L}$.

Conversely, every such matrix defines by (3.6) a functional in $\mathcal{F}_{\rho}^{\perp} \cap \underline{E}^{+\prime}$. Note that (3.8) means in particular that the matrix elements $M_{I, J}$ depend only on the equivalence classes $[I]$, $[J]$ of multiindices.

To construct matrices satisfying (3.7) and (3.8) we use a simple method which we now describe. It goes back to [9] and has been used in a similar context e.g. in [6] and [10]. Suppose we want to construct an infinite, hermitian matrix $A=\left(a_{i j}\right)_{i, j \in N}$ such that

1') $\sum \bar{\lambda}_{i} \lambda_{j} a_{i j} \geq \sum c_{j}\left|\lambda_{j}\right|^{2}$

for all finite sequences $\left\{\lambda_{i}\right\}$, where the $c_{j} \geq 0$ are some given numbers.

$\left.2^{\prime}\right) \quad a_{n n}=a_{i j}$ for certain $i j$ depending on $n$. Consider the matrix $B=\left(b_{i j}\right)_{i, j \in N}$ with $b_{i j}=a_{i j}-\delta_{i j} c_{j}$ and let $B_{n}$ denote the submatrix $\left(b_{i j}\right)_{i, j \leq n}$. Then (3.9) is certainly true if $\operatorname{det} B_{n}>0$ for all $n$. Now

$$
\operatorname{det} B_{n+1}=\operatorname{det} B_{n} \cdot\left(b_{n+1, n+1}-V_{n} \cdot B_{n}^{-1} V^{T}{ }_{n}\right)
$$

with $V_{n}=\left(b_{n+1,1}, \cdots, b_{n+1, n}\right)$. We can therefore define the matrix elements of $B$ by induction over $n$, such that (3.9) and (3.10) hold, provided the constraint (3.10) satisfies the following condition:

If $i j \neq n n$ is such that (3.10) requires $a_{n n}=a_{i j}$ to hold, then either $i>n$ or $j>n$.

In fact, $V_{n}$ will then only involve $b_{k k}$ with $k \leq n$. If det $B_{n}>0$ and the off-diagonal elements not fixed by (3.10) are arbitrarily defined (e.g. as zero), then $\operatorname{det} B_{n+1}>0$ for $b_{n+1, n+1}$ large enough, in virtue of (3.11).

In order to apply this to the problem at hand we must show that it is possible to order the equivalence classes $[I]$ into a sequence, such that whenever $I^{*} I \sim J^{*} K$, then either $[J]$ or $[K]$ appear later in the sequence than $[I]$.

We define such an order relation $<$ by requiring $[I]<[J]$ if $\operatorname{Deg} I<\operatorname{Deg} J$ and choosing an arbitrary linear ordering for classes with fixed degree. This ordering has the right properties:

3.4 Lemma. If $I^{*} I \sim J^{*} K$ and $[I] \neq[J],[I] \neq[K]$, then either $[I]<[J]$ or $[I]<[K]$.

Proof. There are three possibilities:

1) $\operatorname{Deg} I<\operatorname{Deg} J$ which implies $[I]<[J]$.

2) $\operatorname{Deg} I>\operatorname{Deg} J$ which implies $\operatorname{Deg} I<\operatorname{Deg} K$ because 
2Deg $I=\operatorname{Deg} J+\operatorname{Deg} K$, and thus $[I]<[K]$.

3) $\operatorname{Deg} I=\operatorname{Deg} J$ which implies $[I]=[J]$ and $[I]=[K]$ by Lemma 3.2.

By the preceding discussion, we can thus for any choice of $c_{[I]} \geq 0$ define a positive linear functional $T \in \underline{E}^{+\prime} \cap \mathcal{F}_{\rho}^{+}$such that

$$
\sum_{[I]} c_{[I]}\left|\sum_{J \sim I} \alpha_{J}\right|^{2} \leq T\left(a^{*} a\right)
$$

for all $a=\sum \alpha_{I} e_{I}$. The seminorms $a \mapsto\left(\sum_{I} c_{[I]}\left|\sum_{J \sim I} \alpha_{J}\right|^{2}\right)^{1 / 2}$ form a basis for the family of continuous seminorms on $\underline{E}$ which vanish on $\mathscr{F}_{\rho}$. Another way of stating this result is thus:

3.5 Theorem. For any continuous seminorm $\|\cdot\|$ on $\underline{E}$, vanishing on $\mathcal{F}_{\rho}$, there is a $T \in \underline{E}^{+\prime} \cap \mathcal{F}_{p}^{\perp}$ such that $\|a\|^{2} \leq T\left(a^{*} a\right)$ for all $a \in \underline{E}$.

3.6 Corollary. The ideals $\mathscr{F}_{\rho}$ satisfy the conditions stated in Proposition 2.2. Moreover, $\left(E / \mathscr{F}_{p}\right)^{+}$is a normal cone in $\left(\underline{E} / \mathscr{F}_{p}\right)$.

Proof. This follows immediately from the preceding theorem, cf. Thm. 4 in [6]. Note that the product in $\underline{E}$ is continuous in both factors jointly, so the analogue of the topology $N$ in [6] is here the original topology.

\section{§. The Locality Ideal}

The locality ideal $\mathscr{F}_{c} \subset \underline{\mathscr{P}}$ is generated by commutators $f \otimes g-g \otimes f$ with $f, g \in \varphi_{1}$ and $f(x) g(y)=0$ for $(x-y)^{2} \geq 0 . \quad \mathscr{F}_{c}$ is a graded ideal w.r.t. the canonical grading of $\underline{\varphi}$, i.e. if $\underline{f}=\left(f_{0}, \cdots, f_{N}, 0, \cdots\right) \in \mathcal{F}_{c}$, then $f_{n} \in \mathscr{F}_{c} \cap \mathscr{S}_{n}=\mathscr{F}_{c, n}$ for all $n$. $\quad \mathscr{F}_{c}$ is also $*$-invariant and the quotient algebra $A=\underline{\mathscr{S}} / \mathscr{F}_{c}$ is again a graded *-algebra:

$$
A=\bigoplus_{n=0}^{\infty} A_{n}
$$

where $A_{n}:=\mathscr{Y}_{n} / \mathscr{F}_{c, n}$. Equipped with the quotient topology $A$ is a nuclear $L F$-space.

It will be convenient to have a more explicit description of $\mathscr{F}_{c, n}, A_{n}$ and the duals $A_{n}^{\prime} \simeq \mathcal{F}_{c, n}^{\perp}$. To this end we generalize somewhat a construction called "space-like symmetrization" in [1]. Define first an equivalence relation between points in $\boldsymbol{R}^{d \cdot n}$ :

$$
\left(x_{1}, \cdots, x_{n}\right) \sim\left(y_{1}, \cdots, y_{n}\right)
$$

if one $n$-tuple can be transformed into the other by a sequence of transpositions 
of the components, such that one transposes at each step a pair of adjacent components, that are mutually space-like. To simplify the notation we also denote such $n$-tuples by capital letters, $X=\left(x_{1}, \cdots, x_{n}\right)$. If $\pi$ is a permutation of $\{1, \cdots, n\}$ we write $X_{\pi}=\left(x_{\pi 1}, \cdots, x_{\pi n}\right)$. For every $X$ we have a set of permutations:

$$
P_{X}:=\left\{\pi \mid X_{\pi} \sim X\right\}
$$

and

$$
\{Y \mid Y \sim X\}=\left\{X_{\pi} \mid \pi \in P_{X}\right\} .
$$

Note that $P_{X}$ is in general only a subset and not a subgroup of the symmetric group, Moreover, if $Y \sim X$, say $Y=X_{\pi_{1}}$, then

$$
P_{Y}=P_{X} \circ \pi_{1} \text { and } P_{X}=P_{Y} \circ \pi_{1}^{-1} \text {. }
$$

Next we define $2^{n(n-1) / 2}$ open sets $\mathcal{O}_{\sigma} \in \boldsymbol{R}^{d \cdot n}$ as follows: Let $\sigma=\left\{\sigma_{i j}\right\}$ be a set of signs, i.e. $\sigma_{i j}= \pm 1$ for $i, j=1, \cdots, n, i<j$. Then

$$
\mathcal{O}_{\sigma}:=\left\{X \mid\left(x_{i}-x_{j}\right)^{2} \cdot \sigma_{i j}>0 \text { for } i<j\right\} \text {. }
$$

Denote by $\mathscr{Y}\left(\overline{\mathcal{O}}_{\sigma}\right)$ the space of $C^{\infty}$-functions $\mathcal{O}_{\sigma} \rightarrow \boldsymbol{C}$, such that

$$
\|f\|_{k, \mathcal{O}_{\sigma}}:=\max _{\left|\alpha_{i}\right| \leq k} \sup _{X \in \mathcal{O}_{\sigma}}\left|\prod_{i} D^{\omega_{i}} f\left(x_{1}, \cdots, x_{n}\right) \cdot \prod_{j}\left(1+\left|x_{j}\right|^{k}\right)\right|
$$

is finite for all $k$. These seminorms define a topology on $\mathscr{Y}\left(\overline{\mathcal{O}}_{\sigma}\right)$. The sets $\mathcal{O}_{\sigma}$ are regular open sets in the sense of Whitney. It follows (cf. e.g. [12] pp. 77-79) that $\mathscr{Y}\left(\overline{\mathcal{O}}_{\sigma}\right)$ is isomorphic to the quotient space of $\mathscr{S}\left(\boldsymbol{R}^{d \cdot n}\right)$ modulo functions with support in $C \mathcal{O}_{\sigma^{*}}$. The isomorphism is given by restricting functions in $\mathscr{S}\left(\boldsymbol{R}^{d \cdot n}\right)$ to $\mathcal{O}_{\sigma}$. As a consequence of this we have also that the dual space $\mathscr{S}\left(\overline{\mathcal{O}}_{\sigma}\right)^{\prime}$ is isomorphic to a subspace of $\mathscr{Y}\left(\boldsymbol{R}^{d \cdot n}\right)^{\prime}$ namely to the space of tempered distributions with support in $\overline{\mathcal{O}}_{\sigma}$.

If $X \in \boldsymbol{R}^{d \cdot n}$, the set of permutations $P_{X}$ depends only on the sign of the $\left(x_{i}-x_{j}\right)^{2}$. Hence, for $\sigma$ fixed, $P_{X}$ is independent of $X \in \mathcal{O}_{\sigma}$ and we can write $P_{\sigma}$ instead of $P_{X}$. If $\pi \in P_{\sigma}$, define $\pi \sigma$ by $(\pi \sigma)_{i j}=\sigma_{\pi^{-1}, \pi^{-1} j}$. Then

$$
\mathcal{O}_{\pi \sigma}=\left\{X_{\pi} \mid X \in \mathcal{O}_{\sigma}\right\} \text {. }
$$

If $f$ is a function defined on a subset of $\boldsymbol{R}^{d \cdot n}$ and $\pi$ is a permutation, we write

$$
(f \circ \pi)(X)=f\left(X_{\pi}\right) \text {. }
$$

For distributions $T$ we define

$$
(T \circ \pi)(f)=T\left(f \circ \pi^{-1}\right) .
$$


Then $T \circ \pi$ has support in $\overline{\mathcal{O}}_{\sigma}$ if $T$ has support in $\overline{\mathcal{O}}_{\pi \sigma}$ and $\pi \in P_{\sigma}$.

After this preparation we can now state

4.1 Proposition. (i) A distribution $T \in \mathscr{Y}\left(\mathbb{R}^{n^{\circ d}}\right)^{\prime}$ annihilates $\mathscr{F}_{c, n}$ if and only if $T$ can be written as

$$
T=\sum_{\sigma} T_{\sigma}
$$

with supp $T_{\sigma} \in \overline{\mathcal{O}}_{\sigma}$ and $T_{\sigma}=T_{\pi \sigma} \circ \pi$ for all $\pi \in P_{\sigma}$.

(ii) If $T$ runs through an equicontinuous set in $\mathcal{F}_{c, n}^{\perp}$, then the $T_{\sigma}$ can be chosen from equicontinuous sets in $\mathscr{P}\left(\overline{\mathcal{O}}_{\sigma}\right)^{\prime}$.

The proof is an application of standard facts from distribution theory. It is given in the appendix.

From this proposition one obtains a characterization of $\mathscr{F}_{c, n}$ and $A_{n}$. Define for $f \in \mathscr{S}\left(\boldsymbol{R}^{d \cdot n}\right)$

$$
\hat{f}(X):=\frac{1}{\operatorname{Card} P_{X}} \sum_{\pi \in P_{X}} f\left(X_{\pi}\right)
$$

Note that $\hat{f}$ can have discontinuities on $\partial \mathcal{O}_{\sigma} \cap \partial \mathcal{O}_{\sigma^{\prime}}$ if $P_{\sigma} \neq P_{\sigma^{\prime}}$. However, $\hat{f} \mid \mathcal{O}_{\sigma} \in \mathscr{Y}\left(\overline{\mathcal{O}}_{\sigma}\right)$ for all $\sigma$.

4.2 Proposition. (i) $f \in \mathscr{F}_{c, n}$ if and only if $\hat{f}=0$.

(ii) The mappings

$$
f \mapsto \hat{f} \mid \mathcal{O}_{\sigma}
$$

define an isomorphism of $A_{n}$ onto the closed subspace of $\underset{\sigma}{\bigoplus_{\sigma}} \mathscr{Y}\left(\overline{\mathcal{O}}_{\sigma}\right)$ which consists of $\left\{g_{\sigma}\right\}$ satisfying $g_{\sigma}=g_{\pi \sigma} \circ \pi$ for all $\pi \in P_{\sigma}$.

Proof. This is the dual version of Proposition 4.1 and follows by combining it with the bipolar theorem. Note that $\hat{f}=0$ is equivalent to $\sum_{\pi \in P_{\sigma}}(f \circ \pi) \mid \mathcal{O}_{\sigma}=0$ for all $\sigma$.

In order to apply the results of Section 3 to the algebra $A$, we now define homomorphisms of $A$ onto partially symmetric tensor algebras over finite dimensional spaces.

Let $\left\{e_{x}\right\}_{x \in \boldsymbol{R}^{d}}$ denote the natural basis in the (infinite dimensional) vector space $\mathbb{C}^{R^{d}}$. If $x=\left\{x_{1}, \cdots, x_{n}\right\}$ is a finite subset of $\mathbb{R}^{d},\left\{e_{x}\right\}_{x \in x}$ is then a basis for a finite dimensional subspace $E_{\varkappa} \simeq \mathbb{C}^{\varkappa}$. Define a relation on the set $x$ :

$$
\rho_{x}=\left\{\left(x_{i}, x_{j}\right) \mid\left(x_{i}-x_{j}\right)^{2}<0\right\},
$$


and let $\Im_{\varkappa}$ be the corresponding two-sided ideal in the tensor algebra $\underline{E}_{x}$, generated by the commutators $\left[e_{x}, e_{y}\right]$ with $(x-y)^{2}<0$.

If $x \subset y$, we have natural projections $E_{y} \rightarrow E_{x}$ which extend to $*$-homomorphisms $q_{x y}: E_{y} \rightarrow E_{x}$ satisfying

$$
q_{x y} \circ q_{y z}=q_{x z} \text { if } x \subset y \subset z .
$$

From Lemma 3.1 (ii) follows that $\mathscr{F}_{y}$ is mapped onto $\mathscr{F}_{x}$ under $q_{y x}$, which thus induces a $*$-homomorphism $p_{x y}: A_{y} \rightarrow A_{x}$ where $A_{g}$ denotes the quotient algebra $E_{\mathfrak{g}} / \mathscr{F}_{\mathfrak{g}}$. Again we have

$$
p_{x y} \circ p_{y z}=p_{x z} \quad \text { if } x \subset y \subset z .
$$

Consider now the following linear maps $\mathscr{\varphi} \rightarrow E_{\varkappa}$ :

$$
\psi_{x}(f)=\sum f\left(x_{i}\right) e_{x_{i}}
$$

They extend to $*$-homomorphisms $\psi_{x}: \underline{\varphi} \rightarrow \underline{E}_{x}$, and it is clear that $\psi_{x}\left(\mathscr{F}_{c}\right)$ $\subset F_{x}$. The $\psi_{x}$ thus give rise to $*$-homomorphisms $\Phi_{x}: A \rightarrow A_{x}$. The $\Phi_{x}$ clearly satisfy

$$
\Phi_{x}=p_{x y} \circ \Phi_{y} \quad \text { if } x \subset y,
$$

and the family of all $\Phi_{\varkappa}$ separates points in $A$ :

4.3 Lemma. $\bigcap_{x} \operatorname{Ker} \Phi_{x}=\{0\}$.

Proof. Suppose $f \in \mathscr{S}\left(\boldsymbol{R}^{d \cdot n}\right)$ and $\psi_{x}(f) \in \mathscr{F}_{x}$ for all $x$. By Lemma 3.1 (ii) and the definition of $\rho_{x}$ we have

$$
\sum_{X \sim Y} f(Y)=0
$$

for all $X \in \boldsymbol{R}^{d \cdot n}$. Hence $\hat{f}=0$, so $f \in \mathscr{F}_{c, n}$ by Proposition 4.2 (i).

Combining Theorem 3.3, Lemma 4.3 and using (4.4) and (4.6) we obtain

4.4 Theorem. (i) The algebra $A$ has no divisors of zero.

(ii) The only invertible elements in $A$ are multiples of the identity.

(iii) Let $\mathcal{O} \subset \boldsymbol{R}^{d}$ be an open set and $\mathcal{O}^{c}$ its causal complement, $\mathcal{O}^{c}=\left\{x \mid(x-y)^{2}<0\right.$ for all $y \in \mathcal{O}\}$. Let $A(\mathcal{O})$ be the subalgebra of $A$ generated by functions with support in $\overline{\mathcal{O}}$ and $A(\mathcal{O})^{\prime}$ its commutant. Then $A(\mathcal{O})^{\prime}=A\left(\mathcal{O}^{c}\right)$.

Proof. (i) Suppose $a \neq 0, b \neq 0$. By Lemma 4.3 there are $x$ and $y$ s.t. $\Phi_{x}(a) \neq 0$ and $\Phi_{y}(b) \neq 0$. If $z \supset x \cup y$, we have $\Phi_{x}(a)=p_{x \neq} \circ \Phi_{g}(a)$ and $\Phi_{y}(b)=$ $p_{y g} \circ \Phi_{g}(b)$, so $\Phi_{g}(a) \neq 0$ and $\Phi_{g}(b) \neq 0$. Thus $\Phi_{g}(a) \cdot \Phi_{g}(b)=\Phi_{g}(a b) \neq 0$, so 
$a b \neq 0$.

(ii) If $a \in A$ has an inverse in $A_{\text {, then }} \Phi_{x}(a)$ has an inverse in $A_{x}$ for all $x$. Thus $\Phi_{x}(a)=c_{x} \Phi_{x}(1)$ with $c_{x} \in C$, and we have only to show that $c_{x}$ is independent of $x$. But if $y$ is another finite subset of $\mathbb{R}^{d}$ and $z \supset x \cup y$, we have $\Phi_{x}=p_{x z} \circ \Phi_{z}$ and $\Phi_{y}=p_{y z} \circ \Phi_{z}$ and thus $c_{x}=c_{y}=c_{z}$.

(iii) Using Prop. 3.2 we may for every open set $\mathcal{O} \subset \mathbb{R}^{d}$ identify $A\left(\mathcal{O}^{c}\right)_{n}$ with the space of all symmetrized functions $\hat{f}$ with $f \in \mathscr{P}_{n}$ having support in $\mathcal{O}^{c} \times \cdots \times \mathcal{O}^{c}$. This space is obviously closed in $A_{n}$ w.r.t. pointwise convergence of functions. It follows that $a \in A\left(\mathcal{O}^{c}\right)$ iff $\Phi_{x}(a) \in \Phi_{x}\left(A\left(\mathcal{O}^{c}\right)\right)$ for all finite subsets $x \subset \mathbb{R}^{d}$. Now suppose $a \in A(\mathcal{O})^{\prime}$. If $x \subset \mathbb{R}^{d}$ is finite, there is a $y \supset x$, such that $(y \cap \mathcal{O})^{c} \cap y=\mathcal{O}^{c} \cap y$. From Thm. 3.3 (iii) we deduce that $\Phi_{y}(a) \in\left(\Phi_{y}(A(\mathcal{O}))\right)^{\prime}=\Phi_{y}\left(A\left(\mathcal{O}^{c}\right)\right)$, so $a \in A\left(\mathcal{O}^{c}\right)$.

We now come to the question whether $A$ has sufficiently many states. Combining the (continuous) homomorphisms $\Phi_{x}$ with the states on $A_{x}$ we obtain by Corollary 3.6 and Lemma 4.3 a separating family of states on $A$. Hence we have

4.5 Theorem. The locality ideal satisfies the conditions of Prop. 2.2. In particular, the algebra $A$ has a faithful Hilbert space representation.

This result can be strengthened considerably, and the rest of the section will be devoted to this task. Let $\|\cdot\|_{k}, k=1,2, \cdots$ be a basis of continuous seminorms for the topology of $\mathscr{S}\left(\mathbb{R}^{d}\right)$. The seminorms $\|\cdot\|_{k} \otimes_{\varepsilon} \cdots \otimes_{\varepsilon}\|\cdot\|_{k}=$ : $\|\cdot\|_{k}^{n}$ then form a basis for the topology of $\mathscr{S}\left(\mathbb{R}^{d \cdot n}\right)$. A convenient explicit choice for the present purpose is

$$
\|f\|_{k}=\max _{1 \alpha \mid \leq k} \sup _{x \in R^{d}}\left|D^{\infty} f(x) \cdot\left(1+|x|^{k}\right)\right|
$$

which implies

$$
\|f\|_{k}^{n}=\max _{\left|\alpha_{i}\right| \leq k} \sup _{x \in R^{d n}}\left|\prod_{i} D^{\omega_{i}} f\left(x_{1}, \cdots, x_{n}\right) \cdot \prod_{j}\left(1+\left|x_{j}\right|^{k}\right)\right| .
$$

The direct sum topology on $\underline{\mathscr{S}}$ can be defined by the collection of seminorms of the form

$$
\|\underline{f}\|_{\left\{c_{n}\right\},\left\{k_{n}\right\}}=\sup _{n} c_{n}\left\|f_{n}\right\|_{k_{n}}^{n}
$$

with $c_{n} \geq 0$ and $k_{n} \in \mathbb{N}$ for all $n$. A strictly coarser topology, called $\tau_{\infty}$ in [13] and subsequent papers of the Leipzig group, is defined by allowing only bounded sequences $\left\{k_{n}\right\}$ in (4.9). A basis of seminorms for this topology is also given by 


$$
\|\underline{f}\|_{\left.c_{n}\right\}, k}=\sup _{n} c_{n}\left\|f_{n}\right\|_{k}^{n}
$$

with $k \in N$ independent of $n$. By abuse of notation we shall also denote the corresponding quotient topology on $A$ by $\tau_{\infty}$. Some simple properties of this topology are as follows:

1) $\tau_{\infty}$ induces the original topology on $\mathscr{S}_{n}$ resp. $A_{n}$.

2) $\underline{\varphi}\left[\tau_{\infty}\right]$ resp. $A\left[\tau_{\infty}\right]$ is a nuclear space.

3) The multiplication on $\underline{\mathscr{S}}\left[\tau_{\infty}\right]$ resp. $A\left[\tau_{\infty}\right]$ is jointly continuous in both factors.

The announced refinement of Theorem 4.5 is now

\subsection{Theorem. $A^{+}$is a normal cone in $A\left[\tau_{\infty}\right]$.}

We recall (cf. e.g. [14]) that the statement is equivalent to the following: For every $\tau_{\infty}$-continuous seminorm $\|\cdot\|$ on $A$ there is a $\tau_{\infty}$-equicontinuous set of positive functionals, $C^{+} \subset A^{+\prime}$, such that $\|a\| \leq \sup _{T \in C^{+}}|T(a)|$ for all $a \in A$.

Proof of Thm. 4.6. The first step is to reduce the considerations to the homogeneous components $A_{n}$ in $A_{\text {. }}$ Let $\|a\|_{C^{+}}$denote the seminorm $\sup _{T \in C^{+}}|T(a)|$, if $C^{+}$is an equicontinuous set in $A^{+\prime}$. We claim that for any such $C^{+}$and any sequence $\left\{c_{n}\right\}$ of positive numbers there is an equicontinuous set $D^{+} \subset A^{+\prime}$ such that

$$
\sum c_{n}\left\|a_{n}\right\|_{c^{+}} \leq\|a\|_{D^{+}}
$$

for all $a=\sum a_{n}, a_{n} \in A_{n}$. This can be shown in the same way as Lemma 3.2 in [5], with $\|T\|_{c^{+}}=\sup _{\|b\|_{\sigma^{+} \leq 1}}|T(b)|$ replacing the function $F(\omega)$ in [5], or more directly as follows: We have

$$
\sum_{n} c_{n}\left\|a_{n}\right\|_{C^{+}} \leqslant \sup _{n} 2^{n+1} c_{n}\left\|a_{n}\right\|_{c^{+}} \leqslant \sup \left\|\sum_{n} \beta_{n} a_{n}\right\|_{C^{+}},
$$

where the sup is taken over all sequences $\left\{\beta_{n}\right\}$ of real numbers with $\left|\beta_{n}\right| \leqslant$ $2^{n+1} c_{n}$. By a result of Boas [9] we can write $\beta_{n}=\alpha_{n}^{(1)}-\alpha_{n}^{(2)}$ where the $\alpha_{n}^{(i)}$ are sequences of positive type, i.e. such that $\left(\alpha_{n+m}^{(i)}\right)_{n, m}$ is a positive definite matrix. Moreover, an inspection of the proof in [9] shows that the bound $\left|\beta_{n}\right| \leqslant 2^{n+1} c_{n}$ leads to a bound for the $\alpha_{n}^{(i)}$ : There are constants $d_{n}$, depending only on the sequence $\left\{c_{n}\right\}$, such that one can choose $\left|\alpha_{n}^{(i)}\right| \leqslant d_{n}$. Since $T_{\left[\alpha_{n}\right]}:=\left(\alpha_{0} T_{0}, \alpha_{1} T_{1}\right.$, $\cdots$ ) is a positive functional if $T$ is positive and $\left\{\alpha_{n}\right\}$ is a sequence of positive type ([2], Lemma II. 4.3), we conclude that

$$
\sum_{n} c_{n}\left\|a_{n}\right\|_{c^{+}} \leqslant\|a\|_{D^{+}}
$$


with $D^{+}=\left\{S \in \mathscr{S}^{+\prime}\left|S=T_{\left\{\alpha_{n}\right\}}, T \in C^{+},\right| \alpha_{n} \mid \leqslant 2 d_{n}\right\}$.

In view of this it is sufficient to show that for every $k$ there is an equicontinuous set $C^{+} \subset A^{+\prime}$ and a sequence $\left\{c_{n}\right\}$ such that

$$
\left\|a_{n}\right\|_{k}^{n} \leq c_{n}\left\|a_{n}\right\|_{c^{+}}
$$

for all $a_{n} \in A_{n}$. We have here denoted the quotient seminorm corresponding to (4.8) again by $\|\cdot\|_{k}^{n}$. By Proposition 4.2 (ii) we may equivalently consider the seminorms

$$
\max _{\sigma}\left\|\hat{f}_{n}\right\|_{k^{\prime}, \mathcal{O}_{\sigma}}
$$

for some $k^{\prime}$, where the seminorms on $\mathscr{S}\left(\overline{\mathcal{O}}_{\sigma}\right)$ are as in (4.2). In principle $k^{\prime}$ could depend on $n$, but a closer inspection of the proof (which uses extension theorems for $C^{\infty}$-functions, cf. the appendix) shows that one can in fact take $k^{\prime}=k$.

In order to prove (4.11) we generalize the homomorphisms $\Phi_{x}$ slightly. Let $x=\left\{x_{1}, \cdots, x_{N}\right\}$ again be a finite subset of $\mathbb{R}^{d}$ and suppose $k \in \mathbb{N} \cup\{0\}$. Let $E_{x, k}$ be a finite dimensional vector space with basis $\left\{e_{x \alpha}\right\}, x \in x$, $\alpha \in(\mathbb{N} \cup\{0\})^{d},|\alpha| \leq k$. Define $\psi_{x, k}: \varphi \rightarrow E_{x, k}$ by

$$
\psi_{x, k}(f)=\sum_{i=1} \sum_{|\alpha| \leq k} D^{\infty} f\left(x_{i}\right)\left(1+\left|x_{i}\right|^{k}\right) e_{x_{i} \omega} .
$$

In the same way as before this induces homomorphisms $\Phi_{x, k}: \mathscr{S}_{\rightarrow} A_{x, k}:=$ $\underline{E}_{x, k} / \mathscr{F}_{x, k}$, where the ideal $\mathscr{F}_{x, k}$ is generated by $\left[e_{x \alpha}, e_{y \beta}\right]$ with $(x-y)^{2}<0, x$, $y \in x,|\alpha|,|\beta| \leq k$. Now if $a_{n}=f+\mathscr{F}_{c, n} \in A_{n}=\mathscr{Y}_{n} / \mathscr{F}_{c, n}$, we have for any $\varepsilon>0$

$$
\left\|a_{n}\right\|_{k}^{n}-\varepsilon \leq\left|\left(\prod_{i} D^{\bar{\alpha}_{i}} \hat{f}\right)\left(\bar{x}_{1}, \cdots, \bar{x}_{n}\right) \prod_{j}\left(1+\left|\bar{x}_{j}\right|^{k}\right)\right|
$$

for some $\bar{x}_{i} \in \mathbb{R}^{d},\left|\bar{\alpha}_{i}\right| \leq k, i=1, \cdots, n$. By Lemma 3.1 (i) and the definition of $\hat{f}$ we can write the right hand side as

$$
\left|\left(s \circ \Phi_{x, k}\right)\left(a_{n}\right)\right|
$$

where $x:=\left\{\bar{x}_{1}, \cdots, \bar{x}_{n}\right\}$ and $s$ is the linear functional on $A_{x, k}$ taking the value 1 on (the equivalence class of) $e_{\bar{x}_{1} \bar{\alpha}_{1}} \otimes \cdots \otimes e_{\bar{x}_{n} \bar{\alpha}_{n}}$ and 0 on the other basis elements. By Theorem 3.5 we can split $s$ into positive functionals, so

$$
\left|s \circ \Phi_{x, k}\left(a_{n}\right)\right| \leq\left|t \circ \Phi_{x, k}\left(a_{n}\right)\right|
$$

for some positive functional $t$ on $A_{x, k}$. Now $T:=t \circ \Phi_{x, k}$ is a positive functional on $A$ and is continuous w.r.t. the seminorm $\|\cdot\|_{k}^{n}$. Although $s$ and $t$ 
depend on $a_{n}$, they do so only in a trivial way. In fact, the functionals depend only on the particular choice of a basis element in $A_{x, k}$ which one has to make for each $a_{n}$. Changing $a_{n}$ may change the set $x$ and thus the algebra $A_{x, k}$. For fixed $n$, however, there are only finitely many isomorphism classes of such algebras, and for a fixed isomorphism class one has only to consider finitely many basis elements. Altogether there are only finitely many different functionals $s$ and $t$ to be taken into account. It follows that the functionals $T=t \circ \Phi_{x, k}$ belong to an equicontinuous set of positive functionals, independent of $a_{n}$, so we have established (4.11).

From Theorem 4.6 follows that every $\tau_{\infty}$-continuous functional on $A$ is a linear combination of functionals in $A^{+\prime}$. Because the topology $\tau_{\infty}$ induces the original topology on $\bigoplus_{n=0}^{N} A_{n}$ for $N<\infty$, Theorem 4.6 thus has the following corollary:

4.7 Corollary. If $T=\left(T_{o}, T_{1}, \cdots\right) \in \mathcal{F}_{c}^{\perp}$ and $T_{n}=0$ for $n>N$ with $N<\infty$, then $T$ belongs to the linear span of $\mathcal{F}_{c}^{\perp} \cap \mathscr{\varphi}^{+\prime}$.

As already remarked, Theorem 4.6 states that $\tau_{\infty}$ is the topology of uniform convergence on $\tau_{\infty}$-equicontinuous sets in $A^{+\prime}$. Since $A\left[\tau_{\infty}\right]$ is a nuclear algebra, we may equivalently consider order bounded sets in $A\left[\tau_{\infty}\right]^{+/}$:

4.8 Theorem. For every $\tau_{\infty}$-continuous seminorm $\|\cdot\|$ on $A$ there is a $\tau_{\infty}$-continuous, positive functional $T$ on $A$, such that $\|a\|^{2} \leq T\left(a^{*} a\right)$ for all $a \in A$.

Proof. By [16], Prop. 4.1.4, there exists an equicontinuous sequence $\left\{S_{i}\right\}$ in $A\left[\tau_{\infty}\right]^{\prime}$ and a summable sequence $\left\{\lambda_{i}\right\}$ in $\mathbb{R}_{+}$with $\|a\| \leq \sum \lambda_{i}\left|S_{i}(a)\right|$ for all $a$. The $S_{i}$ can be chosen hermitian, and by Theorem 4.6 each $S_{i}$ can be split, $S_{i}=T_{i}^{1}-T_{i}^{2}$, where $\left\{T_{i}^{1}\right\}$ and $\left\{T_{i}^{2}\right\}$ are equicontinuous sequences in $A\left[\tau_{\infty}\right]^{+\prime}$. Hence by Cauchy-Schwarz:

$$
\begin{aligned}
\|a\| \leqq \sum \lambda_{i}\left(\left|T_{i}^{1}(a)\right|+\left|T_{i}^{2}(a)\right|\right) \leq \text { const. } \sum \lambda_{i}\left(T_{i}^{1}\left(a^{*} a\right)^{1 / 2}+T_{i}^{2}\left(a^{*} a\right)^{1 / 2}\right) \\
\leq \text { const. } T\left(a^{*} a\right)^{1 / 2}
\end{aligned}
$$

with $T:=\sum \lambda_{i}\left(T_{i}^{1}+T_{i}^{2}\right)$.

4.9 Concluding remark. $\tau_{\infty}$ is not the strongest topology on $A$ for which $A^{+}$is a normal cone. In fact there exist functionals in $\mathcal{F}_{c}^{+} \cap \underline{\varphi}^{+\prime}$ that are not $\tau_{\infty}$-continuous, cf. Theorem 3.7 in $[5]^{1)}$. The strongest topology for which $A^{+}$

1) There exist even Wightman functionals that are not $\tau_{\infty}$-continuous [17]. Explicit examples can be constructed by taking suitable superpositions of functionals corresponding to free fields with different masses and different normalizations of the field operators. Other examples are provided by generalized Wick powers of generalized free fields (E. Brüning, private communication). 
is normal should have some similarity with the topology $\hat{\tau}$ in [5], but its explicit description seems to be considerably more involved.

\section{Appendix}

Proof of Proposition 4.1. It is obvious that a $T \in \mathscr{Y}_{n}^{\prime}$ having the stated properties annihilates $\mathscr{F}_{c, n}$. Suppose conversely that $T \in \mathscr{F}_{c, n}^{\perp}$. By [15] p. 97 we can decompose $T$ :

$$
T=\sum_{\sigma} T_{\sigma}^{(0)}
$$

with supp $T_{\sigma}^{(0)} \subset \overline{\mathcal{O}}_{\sigma}$. This decomposition is unique only up to terms with support in $\bigcup_{\sigma} \partial \mathcal{O}_{\sigma}$, so the $T_{\sigma}^{(0)}$ need not yet have the required symmetry property. We define their symmetrization by

$$
\hat{T}_{\sigma}^{(0)}=\frac{1}{\operatorname{Card} P_{\sigma}} \sum_{\pi \in P_{\sigma}} T_{\pi \sigma}^{(0)} \circ \pi
$$

Then $\hat{T}_{\pi \sigma}^{(0)} \circ \pi=\hat{T}_{\sigma}^{(0)}$ for all $\pi \in P_{\sigma}$ because of (4.1). If $f$ has support in $\overline{\mathcal{O}}_{\sigma}$, we have moreover $T_{\sigma}^{(0)}(f)=T(f)=(T \circ \pi)(f)=\left(T_{\pi \sigma}^{(0)} \circ \pi\right)(f)$ for $\pi \in P_{\sigma}$. Thus, $T_{\sigma}^{(0)}(f)=\hat{T}_{\sigma}^{(0)}(f)$ for $\operatorname{such} f$, so

$$
T^{(1)}:=T-\sum_{\sigma} \hat{T}_{\sigma}^{(0)}
$$

has support in $\bigcup_{\sigma} \partial \mathcal{O}_{\sigma^{*}} T^{(1)}$ might still be different from zero, because of possible terms with support in $\partial \mathcal{O}_{\sigma} \cap \partial \mathcal{O}_{\sigma^{\prime}}$ for $\sigma, \sigma^{\prime}$ with $P_{\sigma} \neq P_{\sigma^{\prime}}$.

In order to deal with this ambiguity, consider those points $X \in \partial \mathcal{O}_{\sigma}$ which have the same symmetry as $\mathcal{O}_{\sigma}$, i.e. for which $P_{X}=P_{\sigma}$ holds. These are precisely the points $X=\left(x_{1}, \cdots, x_{n}\right)$, where $\left(x_{i}-x_{j}\right)^{2}=0$ for some pairs $i j$ with $\sigma_{i j}=+1$, the sign of the other $\left(x_{i}-x_{j}\right)^{2}$ being the same as for points in $\mathcal{O}_{\sigma}$. Let $B_{\sigma}^{(k)}$ denote the set of those $X \in \partial \mathcal{O}_{\sigma}$ where $\left(x_{i}-x_{j}\right)^{2}=0$ for exactly $k$ such pairs. The following properties are easily verified:

1) $\overline{B_{\sigma}^{(k+1)}} \subset \overline{B_{\sigma}^{(k)}} \subset \overline{B_{\sigma}^{(1)}}=\partial \Theta_{\sigma}$, and $B_{\sigma}^{(k)}=\emptyset$ for some $k \leq n+1$.

2) $\overline{B_{\sigma}^{(k)}} \backslash B_{\sigma}^{(k)} \subset \overline{B_{\sigma}^{(k+1)}}$, and if $\sigma \neq \sigma^{\prime}$, then $\overline{B_{\sigma}^{(k)}} \cap \overline{B_{\sigma^{\prime}}^{(k)}} \subset \overline{B_{\sigma^{\prime \prime}}^{(k+1)}}$ for some $\sigma^{\prime \prime}$.

3) $\overline{B^{(k)}}:=\bigcup_{\sigma} \overline{B_{\sigma}^{(k)}}$ is a closed regular set in the sense of [15], p. 98-100, for all $k$.

Because of 3) we can now decompose $T^{(1)}$ :

$$
T^{(1)}=\sum_{\sigma} T_{\sigma}^{(1)}
$$

wtth supp $T_{\sigma}^{(1)} \subset \overline{B_{\sigma}^{(1)}}=\partial \mathcal{O}_{\sigma}$. The decomposition is unique up to terms with 
support in $\bigcup_{\sigma \neq \sigma^{\prime}} \overline{B_{\sigma}^{(1)}} \cap \overline{B_{\sigma^{\prime}}^{(1)}} \subset \overline{B^{(2)}}$. Suppose $f \in \mathscr{Y}^{\prime}$ is such that supp $f \cap \overline{B_{\sigma}^{(1)}}$ $\subset B_{\sigma}^{(1)}$. We can then write $f=g+h$ with supp $g \cap \overline{B^{(1)}}=\emptyset$ and supp $h$ contained in the open set

$$
U_{\sigma}=\left\{X \mid\left(x_{i}-x_{j}\right)^{2}<0 \text { if } \sigma_{i j}=-1\right\} \text { 。 }
$$

Note that $T^{(1)}$ has support in $\overline{B^{(1)}}$ and $P_{\sigma} \subset P_{X}$ for all $X \in \mathcal{O}_{\sigma}$. Hence we have $T_{\sigma}^{(1)}(g)=\left(T_{\pi \sigma}^{(1)} \circ \pi\right)(g)=0$ and $T_{\sigma}^{(1)}(h)=T_{\pi \sigma}^{(1)} \circ \pi(h)$, and thus $T_{\sigma}^{(1)}(f)=T_{\pi \sigma}^{(1)} \circ \pi(f)$ for all $\pi \in P_{\sigma^{*}}$. Defining

$$
\hat{T}_{\sigma}^{(1)}=\frac{1}{\operatorname{Card} P_{\sigma}} \sum_{\pi \in P_{\sigma}} T_{\pi \sigma}^{(1)} \circ \pi
$$

we conclude that

$$
T^{(2)}=T^{(1)}-\sum_{\sigma} \hat{T}_{\sigma}^{(1)}
$$

has support in $\left.\bigcup_{\sigma} \overline{\left(B_{\sigma}^{(1)}\right.} \backslash B_{\sigma}^{(1)}\right) \subset \overline{B^{(2)}}$. Next we decompose $T^{(2)}$ into terms with support in $\overline{B_{\sigma}^{(2)}}$ and by repeating this procedure we obtain for $k=2,3, \cdots$ distributions $\hat{T}_{\sigma}^{(k)}$ with support in $\overline{B^{(k)}} \subset \partial \mathcal{O}_{\sigma}$ satisfying $\hat{T}_{\pi \sigma}^{(k)} \circ \pi=\hat{T}_{\sigma}^{(k)}$ for $\pi \in P_{\sigma}$ and supp $\left(T-\sum_{k^{\prime} \leq k} \sum_{\sigma} \hat{T}_{\sigma}^{\left(k^{\prime}\right)}\right) \subset \overline{B^{(k+1)}}$. Since $\overline{B^{(k+1)}}=\emptyset$ for $k \geq n$ we finally get the desired decomposition of $T$ :

$$
T=\sum_{\sigma} T_{\sigma}
$$

with $T_{\sigma}=\sum_{k} \hat{T}_{\sigma}^{(k)}$. At each step the decomposition can be done in such a way that an equicontinuous set of distributions is decomposed into equicontinuous sets. This follows from the proofs of the decomposition theorems pp. 98-100 in [15], and we give here only a sketch of the argument. First, if $F \subset \mathbb{R}^{n}$ is a sufficiently regular, closed set (e.g. if $F$ is Lipschitzian) one can by Whitney's extension theorems extend $C^{\infty}$-functions on $F$ (that decrease rapidly at infinity) to functions in $\mathscr{S}$ without increasing their Schwartz-norms by more than a constant factor. Using this, one shows in the same manner as in the proof of theorem XXVII in [15] that every $T \in \mathscr{G}^{\prime}$ with support in $F$ can be written as

$$
T=\sum_{|\alpha| \leq N} D^{a s} \mu_{a}
$$

with tempered measures $\mu_{a}$ having their support in $F$.

Moreover, if $T$ runs through an equicontinuous set, we may take the $\mu_{\infty}$ from an equicontinuous set of tempered measures and also choose $N$ independently of $T$. If $\left\{F_{\nu}\right\}$ is a finite covering of $F$ by closed sets, we can define a 
covering of $F$ by disjoint Borel sets $G_{\nu}$ with $G_{\nu} \subset F_{\nu}$ for all $\nu$. If $\chi_{\nu}$ is the characteristic function of $G_{\nu}$, we have

$$
\mu_{a s}=\sum_{\nu} \mu_{a s} \chi_{\nu}
$$

for all $\alpha$, and $T_{\nu}=\sum_{|\alpha| \leq N} D^{\infty}\left(\mu_{\alpha} \chi_{\nu}\right)$ has support in $\bar{G}_{\nu} \subset F_{\nu}$. This splitting of $T$ obviously respects equicontinuity, and the subsequent symmetrization does not alter this.

\section{Acknowledgements}

I would like to thank Jón Magnússon, E. Brüning and H.J. Borchers for helpful remarks.

\section{References}

[1] Borchers, H.-J., Nuovo Cimento, 24 (1962), 214-236.

[2] — Algebraic aspects of Wightman field theory, Statistical mechanics and field theory, Sen, R., Weil, C. (eds.), New York, Halsted Press, 1972.

[ 3 ] Lassner, G. and Uhlmann, A., Commun. Math. Phys., 7 (1968), 152-159.

[4] Yngvason, J., Commun. Math. Phys., 81 (1981), 401-418.

[ 5] Borchers, H.-J. and Yngvason, J., Commun. Math. Phys., 47 (1976), 197-213.

[6] Yngvason, J., Commun. Math. Phys., 34 (1973), 315-333.

[7] Fritzsche, M., Wiss. Z. Karl-Marx-Univ., 27 (1978), 253-259.

[8] Davis, R.L., Proc. Am. Math. Soc., 4 (1953), 486-495.

[9] Boas, R.P., Bull. Am. Math. Soc., 45 (1939), 399-404.

[10] Schmüdgen, K., Rep. Math. Phys., 10 (1976), 369-384.

[11] Koshmanenko, V.D., Ukr. Mat. Zhurn., 22 (1970), 236-242.

[12] Tougeron, J.C., Ideaux de fonctions differentiables, Berlin, Heidelberg, New-York, Springer 1977.

[13] Lassner, G., On the structure of the test function algebra, JINR preprint, Dubna E 2-5254 (1970).

[14] Schaefer, H.H., Topological Vector Spaces, New-York, Heidelberg, Berlin, Springer 1971.

[15] Schwartz, L., Théorie des distributions, Paris, Hermann 1966.

[16] Pietsch, A., Nuclear locally convex spaces, Berlin, Heidelberg, New-York, Springer 1972.

[17] Hofmann, G., Wiss. Z. Karl-Marx-Univ. Leipzig, Math. -Nat. R., 31 (1982), 27-34. 
